\title{
Sicherheitserhöhung flexibler Handhabungssysteme in der Produktionstechnik durch dezentrale Selbstüberwachung
}

Dipl.-Ing. Andreas Düsel, Dipl.-Ing. Ralf Stöber, Prof. Dr.-Ing. Gerhard Fischerauer Universität Bayreuth, Lehrstuhl für Mess- und Regeltechnik, Universitätsstraße 30, 95447 Bayreuth 0921 55-7231 (Telefon), 0921 55-7235 (Fax), mrt@uni-bayreuth.de

\section{Einleitung}

Flexible, d. h., im Betrieb umkonfigurierbare, mechatronische Systeme (FMS) kommen in immer mehr Anwendungsbereichen zum Einsatz. Verpackungsmaschinen mit FMS können beispielsweise Objekte verschiedener Geometrie verpacken, was eine größere Produktindividualität zulässt [1]. Ebenso werden FMS in der Chirurgie [2], bei der Dekontamination in kerntechnischen Anlagen [3], beim Schleifen von Wafern [4] oder in der Automobilbranche eingesetzt.

Bei den produktionstechnischen Anwendungen bestehen hohe Anforderungen an den Personenschutz und an den Schutz der Werkstücke und Werkzeuge vor Zerstörung. Zunehmend wichtiger werden auch Diagnosemöglichkeiten des FMS hinsichtlich Verschleiß, Fehlererkennung usw. Dies erfordert intelligente Sicherheitseinrichtungen, die Fehlerzustände rechtzeitig erkennen und dadurch Schäden vermeiden helfen. Hier wird am Beispiel eines Werkzeugwechslers für schwere Geräte (z. B. Greifer, Schweißzangen ...) gezeigt, wie sich solche Anforderungen mit möglichst geringem Hard- und Softwareaufwand durch verschiedene ereignisdiskrete Systeme erfüllen lassen. Zudem wird ein Systemkonzept vorgestellt, das es erlaubt, den Verschleißzustand eines solchen FMS im Betrieb zu ermitteln. Damit ließe sich altersbedingten Ausfällen effektiver vorbeugen und die Zuverlässigkeit des Systems erhöhen [5].

\section{Systemarchitekturen}

Als Lösungsansatz zur Erhöhung der Sicherheit von Werkzeugwechslern wurde hier die Strategie verfolgt, eine Selbstüberwachungseinrichtung in den Wechsler selbst zu integrieren Diese dezentrale Integration von Funktionalität wurde gewählt, um dem FMS Eigensicherheit zu verleihen, die unabhängig von der übergeordneten Maschinensteuerung oder der Kommunikationsleitung zu dieser ist.

Es wurden verschiedene Lösungsansätze untersucht und implementiert. Ihnen allen ist die grundlegende Anforderung gemein, dass eine von der Maschinensteuerung angeforderte Werkzeugentriegelung nur dann stattfinden darf, wenn das Werkzeug sich am Ort der vorgesehenen Werkzeugablage befindet. So soll abgesichert werden, dass ein Werkzeug nicht aufgrund eines Bedienungs- oder Programmfehlers am falschen Ort abgeworfen wird, was zu Schäden an Personen oder Maschinen führen kann (z. B. ein Abwurf bei einer hohen Bewegungsgeschwindigkeit). Ein Näherungsschalter liefert die benötigte Information über die Annäherung an die Werkzeugablage. Falls die Werkzeugablage nicht mehr detektiert wird bzw. die Entrieglungsanforderung nicht mehr aufrecht erhalten wird, muss der Werkzeugwechlser seine Verriegelungseinheit wieder verriegeln. Die Ver- und Entriegelung der Werkzeugaufnahme erfolgt über einen pneumatisch betriebenen Kolben. Der Systemstatus wird an die Maschinensteuerung zurückgemeldet (Bild 1).

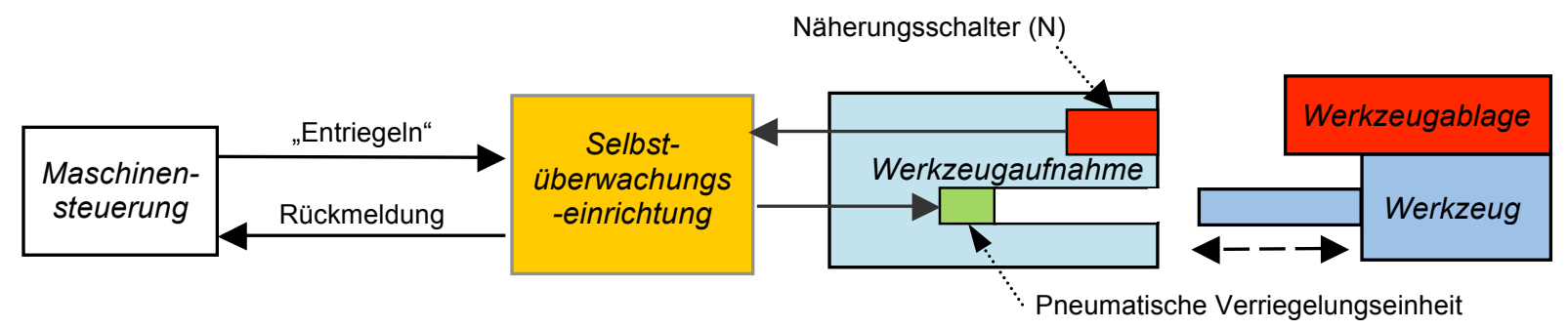

Bild 1: Allgemeine Architektur eines FMS mit dezentraler Selbstüberwachung 


\subsection{Selbstüberwachungseinrichtung mit Relais}

Der Näherungsschalter hat zur Erhöhung der Sicherheit zwei Rückkanäle (Redundanz). Der Kolben soll bei einer Entriegelungsanfrage nur dann entriegeln, wenn beide Kanäle die Anwesenheit der Werkzeugablage signalisieren. Mit den logischen Variablen $A=$ „Entriegelung angefordert“, $N_{1 / 2}=$ „Kanal $1 / 2$ des Näherungsschalters meldet Nähe der Werkzeugablage" und $V=$ "Ventil des pneumatischen Entriegelungsmechanismus ist aktiviert" gilt die Boolesche Gleichung

$$
V=A \cdot N_{1} \cdot N_{2}
$$

Dies lässt sich leicht in Relaistechnik realisieren (Bild 2). Dabei bedeutet $A=1$ physikalisch, dass ein Transistor durchschaltet und der Laststromkreis (für die Versorgung eines Magnetventils zur Steuerung des Pneumatikkreises) mit Spannung versorgt werden. Die beiden Rückkanäle des Näherrungsschalters steuern die beiden abgebildeten Relais. Somit kann erst wenn beide Relais schalten $\left(N_{1}=1\right.$ UND $\left.N_{2}=1\right)$, das Ventil mit Strom versorgt und der Kolben für die Entrieglung bewegt werden. Wenn das Ventil schaltet, wird dies außerdem der Maschinensteuerung rückgemeldet ( $R)$.

Die Vorteile eines Systemaufbaus in dieser Form sind der niedrige Aufwand und die Einfachheit. Es gibt keine Logikbausteine und keine zu programmierenden Controller. Es ist aber auch nicht möglich, ein defektes Magnetventil oder einen defekten Kanal des Näherungsschalters zu detektieren. Bei einer Störung muss manuell geprüft werden, was die Ursache ist. Das System erfüllt somit bei geringem Aufwand die grundlegende Anforderung („Entriegelung nur in der Nähe der Werkzeugablage zulassen“), bietet aber keine zusätzliche Funktionalität.
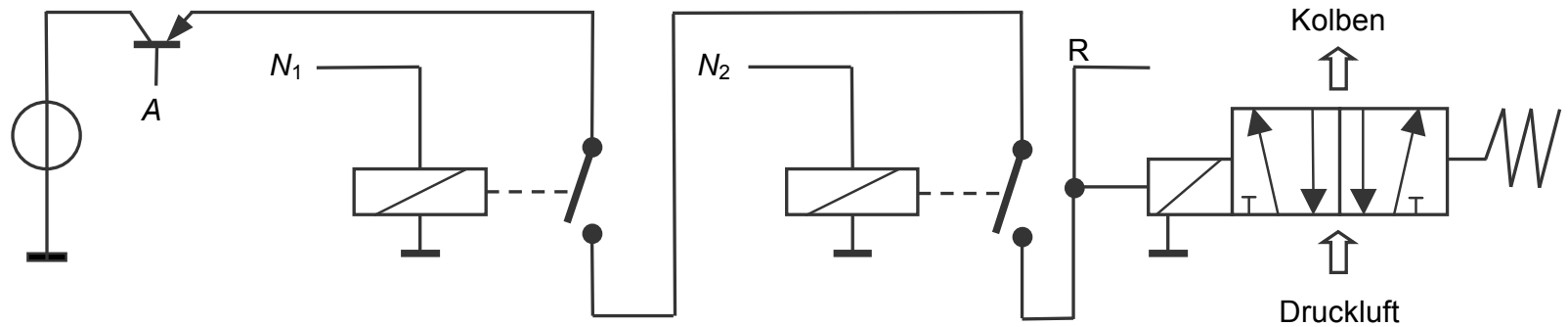

Bild 2: Realisierung von Gleichung (1) mit Relais

\subsection{Selbstüberwachungseinrichtung mit Mikrocontroller}

Die Boolesche GI. (1) lässt sich natürlich auch mit Halbleiter-Treiberstufen in Kombination mit einem Mikrocontroller realisieren. Meldet der Näherungsschalter die Nähe der Werkzeugablage, schaltet eine erste Treiberstufe, so dass eine nachfolgende Treiberstufe an die Versorgungsspannung gelegt wird. Der Mikrocontroller schaltet im Falle einer anliegenden Entriegelungsanforderung die zweite Treiberstufe, welche das Magnetventil aktiviert und somit das Werkzeug entriegelt. Falls nur eine Freigabe anliegt, erfährt der Mikrocontroller dies, da kontinuierlich der Ausgang der ersten Treiberstufe abgefragt wird.

Sollte im Betrieb eine Störung von außen auftreten (z. B. induktive Spannungseinkopplung) und der Mikrocontroller deshalb unzulässig das Magnetventil schalten wollen, so bleibt dies ohne Wirkung, da die hierfür nötige Treiberstufe nur mit Spannung versorgt wird, wenn der Näherungsschalter die Nähe der Werkzeugablage meldet. Eine Einkopplung könnte somit ein unzulässiges Entriegeln schlimmstenfalls nur in unmittelbarer Nähe der Werkzeugablage auslösen.

Zur Erhöhung der Sicherheit werden auch hier wieder beide Kanäle des Näherungsschalters verwendet. Der zweite Kanal versorgt dabei ein zusätzliches (in Reihe geschaltetes) Magnetventil, so dass bei einer Fehlfunktion eines Magnetventils (fehlerhaftes Öffnen ...) das Werkzeug noch nicht entriegelt wird. Der zweite Kanal ist ähnlich wie der erste mit zwei Treiberstufen ausgeführt (Bild 3).

Bei einer Entriegelungsanforderung wird erst eine definierte Zeit gewartet, bis die Treiberstufe geschaltet wird. Dadurch kann ein Fehlimpuls aufgrund von z. B. Induktionsspannungen auch dann kein unbeabsichtigtes Entriegeln auslösen, wenn sich das Werkzeug in der Nähe der Werkzeugablage befindet.

Es versteht sich von selbst, dass ein mikrocontrollerbasiertes System einen höheren Aufwand bedeutet als eine einfache Relaissteuerung wie in Abschnitt 2.1. Allerdings erhöht sich dabei die Sicherheit (zweites Magnetventil, Zeitverzögerung zwischen Entriegelungsanforderung und Schaltvorgang) und es ergeben sich bessere Diagnosemöglichkeiten. Der Mikrocontroller überwacht die Ausgänge der Treiberstufen $\left(R_{1}\right.$ bis $\left.R_{4}\right)$, so dass zum Beispiel bei einer Entrieglungsanfrage, einer vorliegenden Rückmeldung 


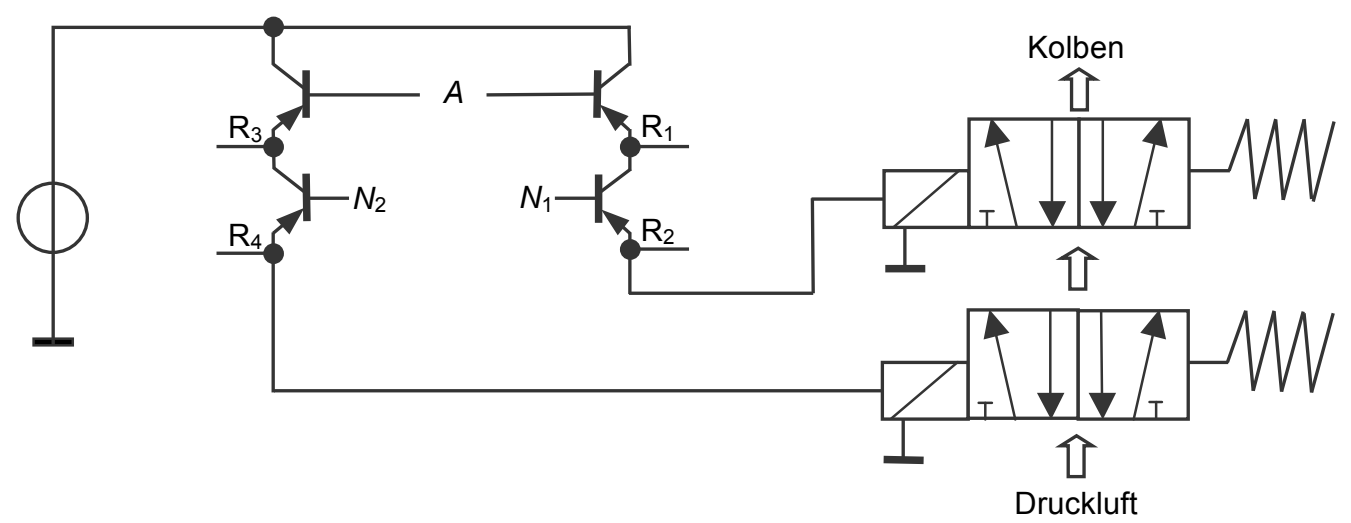

Bild 3: Realisierung von Gleichung (1) mit Transistoren und einem zweiten Magnetventil

über die Näherung zur Werkzeugablage und einer fehlenden Schaltspannung für das Magnetventil gefolgert werden kann, dass die zweite Treiberstufe mit hoher Wahrscheinlichkeit defekt ist.

\subsection{Selbstüberwachungseinrichtung mit Mikrocontroller und zusätzlichen Sensoren}

Dieses System ähnelt dem aus Abschnitt 2.2 - der Unterschied ist, dass noch zwei weitere Endschalter und ein Druckschalter implementiert wurden. Die Endschalter liefern Informationen darüber, ob die pneumatische Verriegelungseinheit ent- oder verriegelt ist. Der Druckschalter meldet, ob der Verriegelungsdruck aufgebaut ist oder nicht. Auch hier ist ein Mikrocontroller die Zentraleinheit und es sind zwei Treiberstufen für jeden Kanal des Endschalters „Näherung“ vorhanden.

In der Signalüberwachungseinrichtung (Bild 4) laufen die Signale der Sensoren sowie die Befehle der Steuerung zusammen. In Abhängigkeit von diesen Signalen gibt das Modul die Befehle zur Ver- und Entriegelung der Werkzeugaufnahme und erkennt Fehlerzustände. Auf dem Mikrocontroller läuft ein Zustandsautomat mit fünf Zuständen (Bild 5).

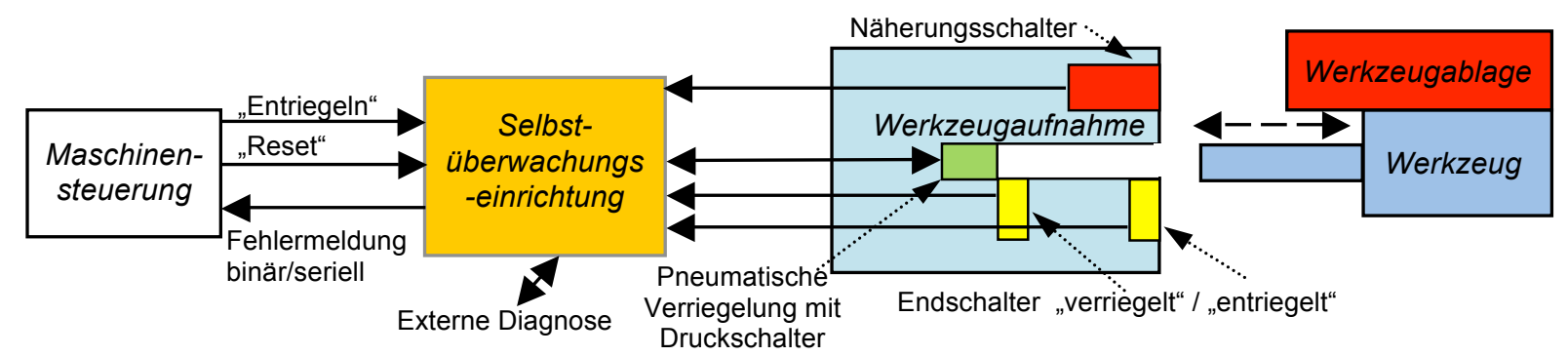

Bild 4: Erweiterte Architektur eines FMS mit dezentraler Selbstüberwachung

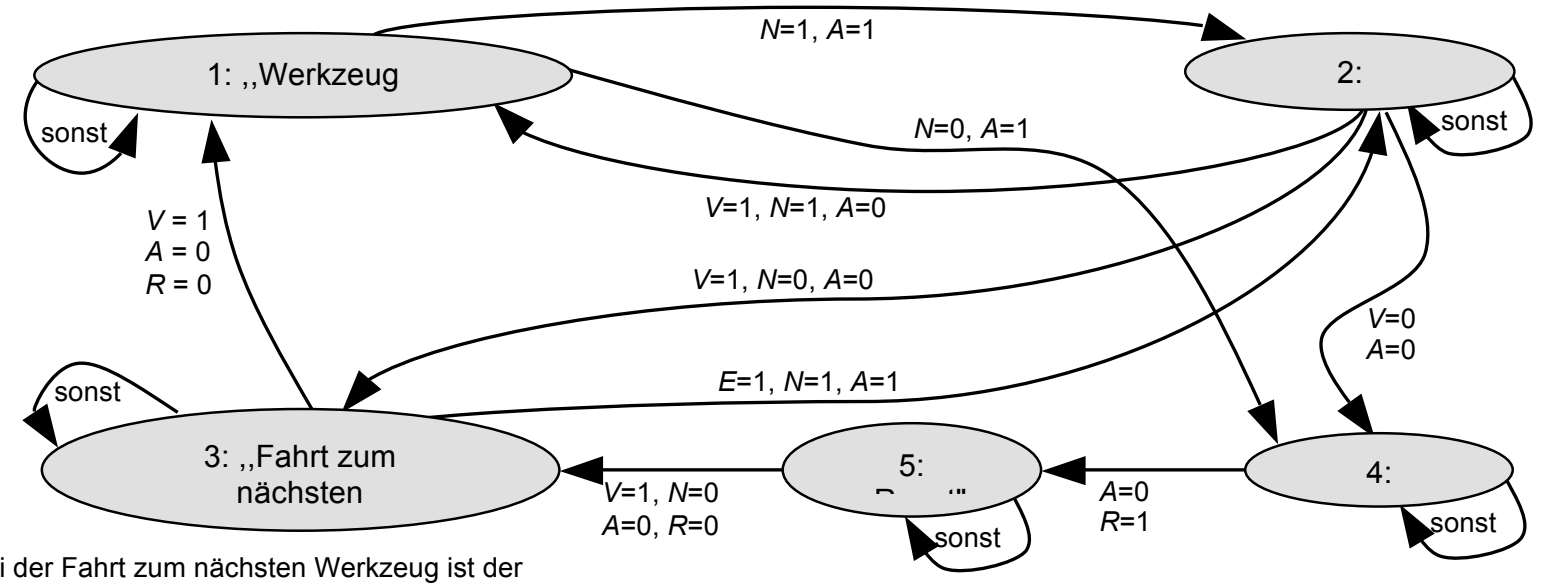

* Bei der Fahrt zum nächsten Werkzeug ist der Werkzeugwechsler ohne Werkzeug verriegelt.

Bild 5: Zustandsautomat für die dezentrale Selbstüberwachung eines Werkzeugwechslers. Die logischen Variablen haben folgende Bedeutung: $E / V=$ „pneumatischer Verriegelungsmechanismus ent- / verriegelt“; $A=$ „Entriegelung angefordert; $N=N_{1} \cdot N_{2}=$,Werkzeugablage ist nahe“; $R=$ „Rücksetzbefehl gegeben“. 
Tabelle 1: Ausgewählte Sicherheitsabfragen in Zustand 1 des Automaten aus Bild 5

\begin{tabular}{|c|c|c|c|c|c|c|c|}
\hline \multirow{2}{*}{ Fall } & \multicolumn{3}{|c|}{ Sensorsignale } & \multicolumn{2}{|c|}{ Steuerbefehle } & \multirow{2}{*}{$\begin{array}{l}\text { Übergang in } \\
\text { Zustand Nr. }\end{array}$} & \multirow{2}{*}{$\begin{array}{l}\text { Sofortiger } \\
\text { Übergang? }\end{array}$} \\
\hline & $E$ & $V$ & $N$ & $A$ & $R$ & & \\
\hline (1) & 0 & 1 & 0 & 1 & 0 & 4 (Fehler) & ja \\
\hline (2) & 0 & 0 & 1 & 1 & 0 & 4 (Fehler) & nein \\
\hline (3) & 1 & 0 & 1 & 1 & 0 & 2 (Entriegelt) & ja \\
\hline
\end{tabular}

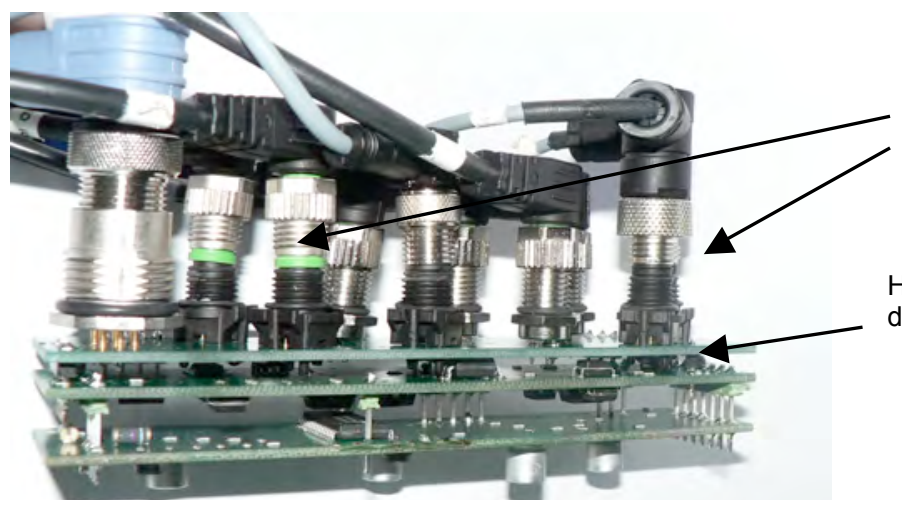

Anschlüsse für Sensoren, Spannungsversorgung und Kommunikation mit der Maschinensteuerung

Bild 6: Selbstdiagnoseeinrichtung im Laborversuch

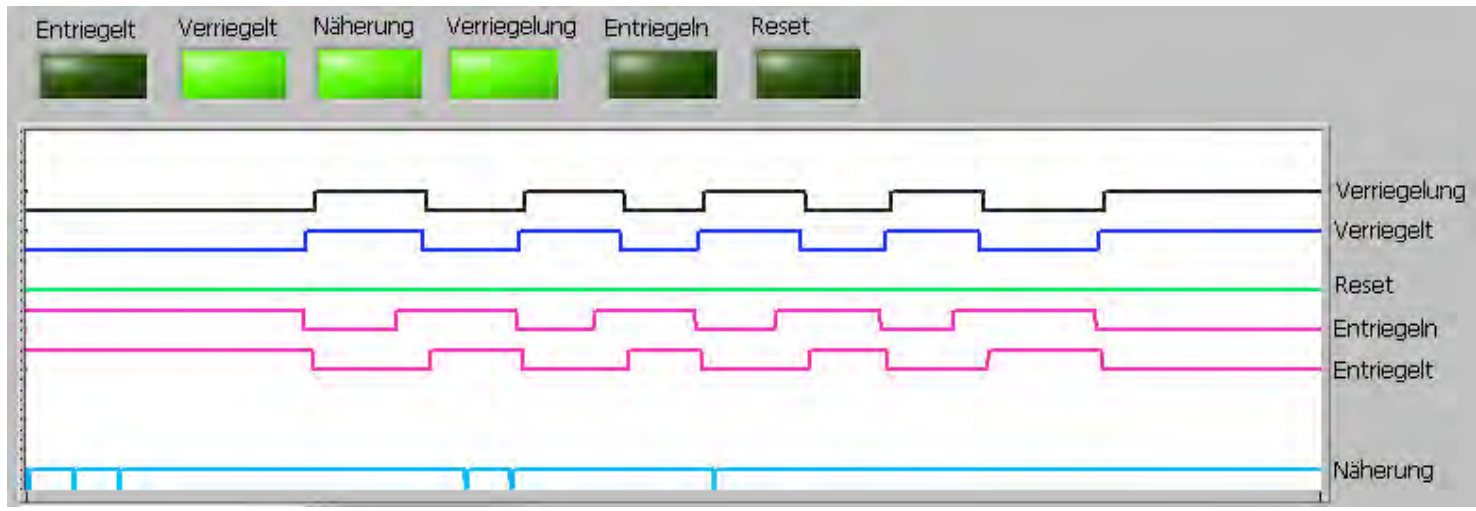

Bild 7: Logikanalyse beim Verriegeln und Entriegeln des Werkzeugs

Nach dem Einschalten befindet sich der Automat in Zustand 5 (Reset). Bevor ein Befehl der Steuerung ausgeführt wird, wird geprüft, ob dies die Sicherheit gefährdet, etwa weil eine Entriegelung außerhalb der Werkzeugablage angefordert wurde $(N=0)$, was natürlich einen unkontrollierten Werkzeugabwurf zur Folge hätte. In einem solchen Fall wird die Ausführung des Entriegelungsbefehls unterdrückt und ein Fehler an die Steuerung gemeldet (Fall (1) aus Tabelle 1).

Fall (2) in Tabelle 1 tritt regelmäßig kurzzeitig beim Entriegeln auf, wenn sich der pneumatische Verriegelungsmechanismus aufgrund seiner mechanischen Trägheit zwischen den Punkten „verriegelt“ und „entriegelt" befindet. Dieser transiente Zustand wird eine bestimmte Zeit toleriert, bevor der Automat in Zustand 4 (Fehler) wechselt, weil der Verriegelungsmechanismus offenbar blockiert.

Führt ein angeforderter Befehl zu keinem sicherheitsgefährdenden Zustand, wird er umgesetzt und der Zustand des Automaten ändert sich entsprechend. So stellt Fall (3) in Tabelle 1 den regulären Abschluss eines Entriegelungsvorgangs dar, bei dem sofort in den Zustand 2 (Entriegelt) übergegangen wird.

Die Selbstüberwachung des betrachteten Werkzeugwechslers wurde an einem Demonstrator in einer produktionsnahen Umgebung untersucht (Bild 6). Dabei wurde die Funktionalität vor allem im Hinblick auf die Sicherheit von Mensch, Maschine und Produktion getestet. Neben Fehlern in der Maschinensteuerung, die normalerweise zum Werkzeugabwurf führen würden, wurden auch Fehler in der Hardware wie der Defekt von Sensoren simuliert. Mit Hilfe einer Logikanalyse konnte das korrekte Verhalten der Selbstüberwachung auch unter Berücksichtigung des Zeitablaufs verifiziert werden (Bild 7).

Über diese um Endschalter und Drucksensor ergänzte Architektur lassen sich auch Defekte an den Näherungssensoren oder an den Ventilen des pneumatischen Verriegelungsmechanismus auffinden. 
Dieses System hat einen weiter erhöhten Aufwand, da zusätzliche Sensoren zum Einsatz kommen. Der Vorteil ist, dass viele Ursachen von Fehlern genau erkannt werden können. Der absolute Aufwand bleibt aber weiterhin gering, da nach wie vor nur wenige Bauelemente (ein kleiner Mikrocontroller, einige Optokoppler, Schaltstufen, Spannungsregler ...) eingesetzt werden.

\subsection{Konzept für eine In-situ-Zustandsdiagnostik}

Die bisher gezeigten Systeme haben die Sicherheit beim Arbeiten mit dem FMS als Ziel. Außerdem können in gewissem Umfang Defekte der eingesetzten Sensoren erkannt werden. Eine Selbstüberwachung des eigentlichen FMS im Bezug auf den Verschleiß und eine Abschätzung der restlichen Lebenserwartung wie in $[6,7]$ ist damit allerdings nicht möglich. Die hierfür erforderliche Zustandsdiagnostik benötigt weitere Sensoren. Derzeit ist noch offen, inwieweit die Zustandsdiagnostik bei Werkzeugwechslern mit mehreren Millionen Wechselzyklen möglich und wirtschaftlich sinnvoll ist. Es bedürfte wohl eines Systems mit z. B. Beschleunigungssensoren, Dehnungsmessstreifen und linearen Positionssensoren in Verbindung mit einer langfristigen Datenspeicherung (Bild 8). Ein entsprechendes System befindet sich im Aufbau, und mittels der daran gewonnen Daten sollen die Vor- und Nachteile des Konzepts untersucht werden. Besondere Aufmerksamkeit muss man der Frage widmen, ob der Einsatz von Mikrosensoren und von Mikroelektronik in einem bisher von der Elektromechanik geprägten Anwendungsfeld die Systemzuverlässigkeit auch wirklich erhöht (und nicht etwa durch den Ausfall der zusätzlichen Baueinheiten erniedrigt - ein aus der Automobiltechnik nur allzu bekanntes Szenario).

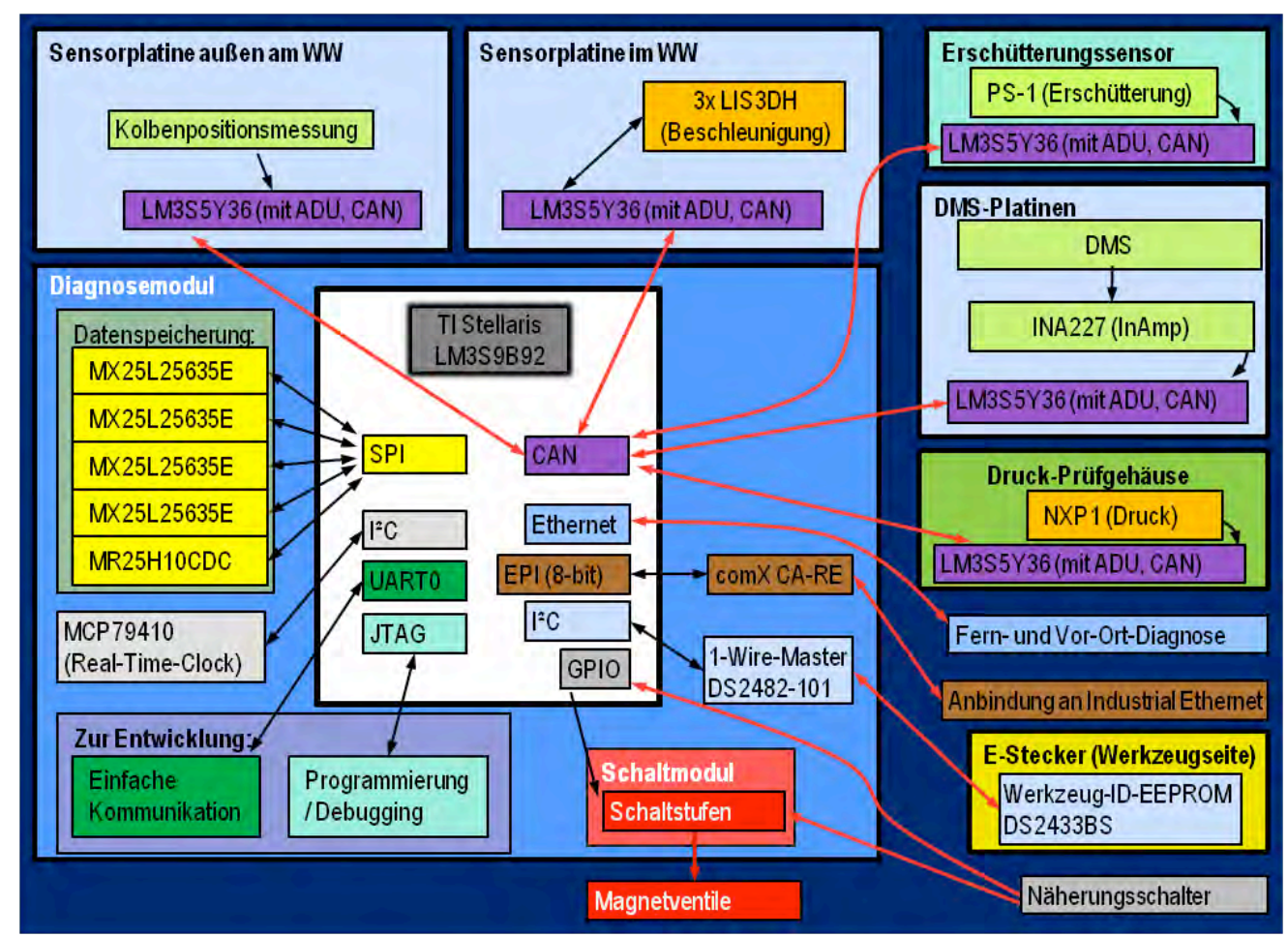

Bild 8: Architektur eines Zustandsdiagnose-Systems für Werkzeugwechsler und andere FMS

\section{Zusammenfassung}

Eine neu konzipierte Sicherheitseinrichtung für Werkzeugwechsler erfasst ohne hohen Aufwand mit Hilfe von Endschaltern den Momentanzustand des Wechselsystems und entscheidet an Hand der Signale, ob eine Entriegelung zulässig ist oder ein Fehler an die Maschinensteuerung gemeldet werden muss. In den experimentellen Untersuchungen konnte nachgewiesen werden, dass Fehlbedienungszustände und Hardwarefehler sicher erkannt werden. Damit lassen sich Personenschäden während der Einrichtung der Anlagen und Schäden an den Werkstücken während der Produktion verhindern. Dieser Sicherheitsgewinn wurde mittlerweile auch schon unter industriellen Bedingungen demonstriert. Aktuell wird das System um zusätzliche Sensoren ergänzt, um auch Aussagen über den Erhaltungszustand des FMS treffen zu können. 


\section{Literatur}

[1] C. Connolly, „Robots at the heart of Schubert packaging machinery lead to great flexibility“, Industrial Robot (2007), Nr. 34, S. 277-280.

[2] R. Michelini, R. Razzoli, „Co-operative minimally invasive robotic surgery“, Industrial Robot (2008), Nr. 35, S. 347-360.

[3] Ch. Kapoor, D. Tesar, „Integrated teleoperation and automation for nuclear facility cleanup“, Industrial Robot (2006), Nr. 33, S. 469-484.

[4] B. Ryuh, „An automatic tool changer and integrated software for a robotic die polishing station“, Mechanism and Machine Theory (2006), Nr. 41, S. 415-432.

[5] X. Zhou, L. Xi, J. Lee, „Reliability-centered predictive maintenance scheduling for a continuously monitored system subject to degradation“, Reliability Engineering and System Safety (2007), Nr. 92, S. 530-534.

[6] A. Heng et al., „Rotating machinery prognostics: State of the art, challenges and opportunities“, Mechanical Systems and Signal Processing (2009), Nr. 23, S. 724-739.

[7] S. J. Bae, W. Kou, Paul V. Kvam, „Degradation models and implied lifetime distributions“, Reliability Engineering and System Safety (2007), Nr. 92, S. 601-608. 